\title{
Quantitative Analysis of Raman Signal Enhancement from Aqueous Samples in Liquid Core Optical Fibers
}

\author{
DAHU QI and ANDREW J. BERGER* \\ The Institute of Optics, University of Rochester, Rochester, New York 14627
}

\begin{abstract}
Raman scattering from aqueous liquids can be collected with high efficiency by enclosing the liquid within a suitable waveguide, as several groups have reported. Here, we present a quantitative model that predicts the relative strength of signals collected from (a) a tubular waveguide and (b) a flat-walled cuvette. Experimental measurements of Raman scattering from aqueous ethanol are made using two geometries, a Teflon ${ }^{\circledR}$-AF waveguide and a standard quartz cuvette. The model correctly predicts the enhancements in several ethanol Raman bands provided by the waveguide geometry. This model should be useful in aligning and characterizing liquid core waveguides, whose manufacture is still undergoing refinements. In particular, the model shows that absorption and scattering losses affect the enhancement factor in different ways.

Index Headings: Raman; Spectroscopy; Teflon; Waveguide; Enhancement.
\end{abstract}

\section{INTRODUCTION}

Vibrational Raman spectroscopy can provide a wealth of information about the chemicals present in a sample. Because Raman scattering is a weak optical effect, it is important to select an efficient excitation/collection geometry. A common method for studying liquids is to place them in a 1-cm-path-length cuvette and irradiate from the side. While this arrangement is often sufficient, special excitation/collection geometries are sometimes employed to increase the signal strength. One idea that dates back to the $1970 \mathrm{~s}^{1}$ is to place the sample in the core of a waveguide, confining the excitation and some of the emission within its guided modes. The apparatus has been variously called a liquid core waveguide (LCW) or, if it is thin and flexible, a liquid core optical fiber (LCOF). In such an approach, emission throughout the sample volume is guided to a common aperture, where it can be conveniently collected. This technique creates much longer excitation and collection zones (overlapping for many $\mathrm{cm}$ or $\mathrm{m}$ ) and requires much smaller volumes $(\mu \mathrm{L})$ than the standard cuvette geometry.

Many liquids of biological interest (e.g., urine, sweat, tears, blood serum) are optically clear and can form the core of an LCW. Due to their aqueous composition, however, a waveguiding material with index of refraction $n$ $<1.33$ is required for the walls of the waveguide. The development of Teflon ${ }^{\circledR}$-AF $(n=1.29)$ by DuPont in the 1990s produced, for the first time, a convenient solid material of suitable refractive index, from which flexible capillary tubes could be formed. Since its introduction, several groups have used Teflon ${ }^{\circledR}$-AF LCWs to obtain Raman signal strengths from aqueous solutions; ${ }^{2-7}$ details

Received 23 July 2003; accepted 17 June 2004.

* Author to whom correspondence should be sent. E-mail: ajberger@ optics.rochester.edu. of some of the enhancements obtained are listed in Table I. The studies report signal enhancements of 20-500 (at excitations of $532 \mathrm{and} /$ or $785 \mathrm{~nm}$ ) as compared to conventional cuvette- or vial-based geometries, indicating the utility of the approach.

To date, the reported Raman enhancement factors from LCWs have not been explicitly compared to theoretical predictions. Altkorn et al. have analyzed the relative performance of different LCW geometries, ${ }^{8}$ but to our knowledge nobody has directly analyzed the absolute advantage of the LCW versus a cuvette geometry. In this paper, we present an analytical model that predicts the relative strength of Raman signals collected from a Teflon ${ }^{\circledR}$-AF waveguide and a cuvette. We also present experimental data for a particular fiber and cuvette and show that the measured enhancement agrees with the theoretical prediction. The model furthermore distinguishes between absorption losses due to the liquid and scattering losses due to the LCW surface; this is useful in determining the quality of a particular LCW.

\section{OPTICAL SYSTEM}

The optical system for obtaining Raman spectra is depicted in Fig. 1. Laser light at $830 \mathrm{~nm}$ (Process Instruments, Salt Lake City, UT) is focused by lens L1 ( $f=$ $35 \mathrm{~mm}, D=25.4 \mathrm{~mm}$ ) onto one end of a $17-\mathrm{cm}$ Teflon AF tube (600 $\mu \mathrm{m}$ inner, $800 \mu \mathrm{m}$ outer diameter), which serves as the liquid core optical fiber (LCOF). The tube was manufactured by Biogeneral, Inc., and was used as received. A syringe injects aqueous samples into the LCOF via plastic tubing and a T-connector; after traversing the LCOF, the liquid flows through a narrow groove to an exit port. Backscattered light from the waveguide is recollimated by lens L1, long-pass filtered (Chroma, Burlington VT), and focused by lens L2 ( $f=60 \mathrm{~mm}, D$ $=25.4 \mathrm{~mm}$ ) into a 12-around-6-around-1 fiber bundle (FiberGuide, Stirling, NJ) composed of 100/140 $\mu \mathrm{m}$ core/ cladding diameter fibers $(\mathrm{NA}=0.22)$. The total diameter of the collection bundle is $700 \mu \mathrm{m}$. At the opposite end of the bundle, the fibers are stacked in a linear array at the entrance slit to a spectrograph $(f / 1.8$ Kaiser Holospec, Ann Arbor, MI) and open-electrode charge-coupled device detector (CCD, Andor Technology, South Windsor, CT). The system also allows substitution of a standard 1-cm-path-length quartz cuvette (wall thickness 1 $\mathrm{mm}$ ) in place of the LCOF, with the excitation beam coming to focus halfway through the cuvette.

Excitation light that traverses the entire LCOF passes through the T-connector, enters a solid optical fiber (OF), and arrives at a power meter (PM). These elements, not involved in Raman measurements, are useful during sys- 
TABLE I. Details from previous experiments in which LCOF-based Raman enhancements were reported. (ID) inner diameter of LCOF tube; (P) power.

\begin{tabular}{|c|c|c|c|c|c|c|c|}
\hline ID $(\mu \mathrm{m})$ & $\begin{array}{l}\text { Length } \\
(\mathrm{cm})\end{array}$ & $\lambda_{\mathrm{ex}}(\mathrm{nm})$ & $\mathrm{P}(\mathrm{mW})$ & Aqueous sample & $\begin{array}{c}\text { Geometry } \\
\left({ }^{\circ}\right)\end{array}$ & $\begin{array}{l}\text { Enhancement } \\
\text { relative to }\end{array}$ & Reference \\
\hline 500 & 100 & 785 & 40 & $\mathrm{NaCO}_{3}$ & 180 & $20 ; 1 \mathrm{~cm}$ cuvette & 2 \\
\hline 50 & 121.5 & 532 & 100 & Lysozyme solution & 180 & $500 ; 1 \mathrm{~cm}$ cuvette & 3 \\
\hline 50 & 100 & 785 & 60 & $10 \%$ 2-propanol & 180 & $\sim 80$; glass vial & 4 \\
\hline 50 & 92 & 532 & 25.7 & $60 \mathrm{mM}$ phenylalanine & 180 & $91 ; 3.8 \mathrm{~cm}$ deep vial & 5 \\
\hline 50 & 41 & 785 & 86.5 & $60 \mathrm{mM}$ phenylalanine & 180 & 58; vial & 5 \\
\hline
\end{tabular}

tem alignment to ensure optimal coupling into the waveguide.

The LCOF holder, shown in detail in Fig. 2, presses a quartz plate (thickness $2 \mathrm{~mm}$ ) against a teflon slab with two holes (for the LCOF and a piece of plastic tubing) connected by a groove. For Raman measurements, liquid enters via the LCOF, flows along the groove, and exits via the tubing. An O-ring provides a liquid-tight seal.

\section{THEORY}

Acceptance Angle. Raman scattering will be emitted isotropically into $4 \pi$ steradians within the aqueous samples considered here. Only a fraction of the emission will lie within the cone angle $\gamma$ that is eventually accepted by the optical system. In the setup shown in Fig. 1, three elements serve as limiting apertures: lens L1, lens L2, and the fiber bundle. As a function of depth, the acceptance angles $\gamma$ (defined within the sample, not in air) for light emitted from points on the optical axis are:

$$
\begin{aligned}
\gamma_{\mathrm{FB}}(z) & =\arctan \left(\frac{f_{1} r_{\mathrm{FB}}}{f_{2} z}\right) \\
\gamma_{\mathrm{L} 1} & =\arctan \left(\frac{r_{1}}{z+n_{\mathrm{w}} f_{1}}\right) \\
\gamma_{\mathrm{L} 2} & =\arctan \left[\frac{f_{1} r_{2}}{z\left(z_{1,2}-f_{1}\right)-n_{\mathrm{w}} f_{1}^{2}}\right]
\end{aligned}
$$

where $z$ is the distance along the optical axis (increasing away from lens L1), $z=0$ is the focal plane of lens L1, $r$ and $f$ are the radii and focal lengths of the various optical elements, respectively, $n_{\mathrm{w}}$ is the refractive index of water, and $z_{1,2}=25 \mathrm{~cm}$ is the distance between the two lenses. The acceptance angle of the system at any depth is the smallest of these three:

$$
\gamma_{\mathrm{sys}}(z)=\min \left(\gamma_{\mathrm{FB}}, \gamma_{\mathrm{L} 1}, \gamma_{\mathrm{L} 2}\right)
$$

This function is plotted for our system in Fig. 3. The influence of the finite thickness of the quartz interface between the sample and the air has been neglected.

All light collected from the waveguide emerges at $z=$ 0 and is therefore viewed with an acceptance angle of $\gamma_{\text {sys }}(0)=14.3^{\circ}$, which by design is slightly greater than the critical angle, $\gamma_{0}=13.9^{\circ}$, of the waveguide. As such, the net acceptance angle for Raman scattering within the waveguide is $\gamma_{0}$, independent of $z$. In contrast, Raman scattering from different depths within the cuvette experiences very different collection angles, as depicted in Fig. 3.

Raman Collection from Waveguide. Mono-exponential Decay Model. For the backscattering geometry em- ployed here, Altkorn et al. have derived an expression for the Raman power emitted from the waveguide due to emissions at various depths $z .^{8}$ It can be written as a Raman power per unit depth, $p_{\mathrm{R}}(z)$ :

$$
p_{\mathrm{R}}(z)=P_{0} s \pi \gamma_{0}^{2}\left[T_{1}(z) \cdot T_{2}(z)\right]
$$

where $P_{0}$ is the excitation power entering the waveguide, $s$ is the Raman scattering probability $\left(\mathrm{cm}^{-1} \mathrm{sr}^{-1}\right), \gamma_{0}$ is the critical angle within the waveguide, and $T_{1}(z)$ and $T_{2}(z)$ are transmission efficiencies for light traveling an axial distance $z$ through the waveguide at the excitation and Raman-emitted wavelengths, respectively. The smallangle approximation $\gamma_{0} \approx \sin \gamma_{0}$ and the meridional-ray approximation are used in the derivation. Total emitted Raman power is simply the integral of $p_{\mathrm{R}}$ over the total length of the waveguide.

When the transmission efficiencies are modeled as mono-exponential decay functions of the form $T_{1}(z)=$ $e^{-\delta_{1} z}, T_{2}(z)=e^{-\delta_{2} z}$, Altkorn et al. show that Eq. 3 predicts a total emitted Raman power of

$$
P_{\mathrm{R}}=P_{0} \pi s \gamma_{0}^{2} \frac{1-e^{-\left(\delta_{1}+\delta_{2}\right) L}}{\delta_{1}+\delta_{2}}
$$

where $L$ is the length of the waveguide. Later in this paper, we will compare the predictions of Eq. 4 to those of a model that treats the $T(z)$ functions as multi-exponential decays.

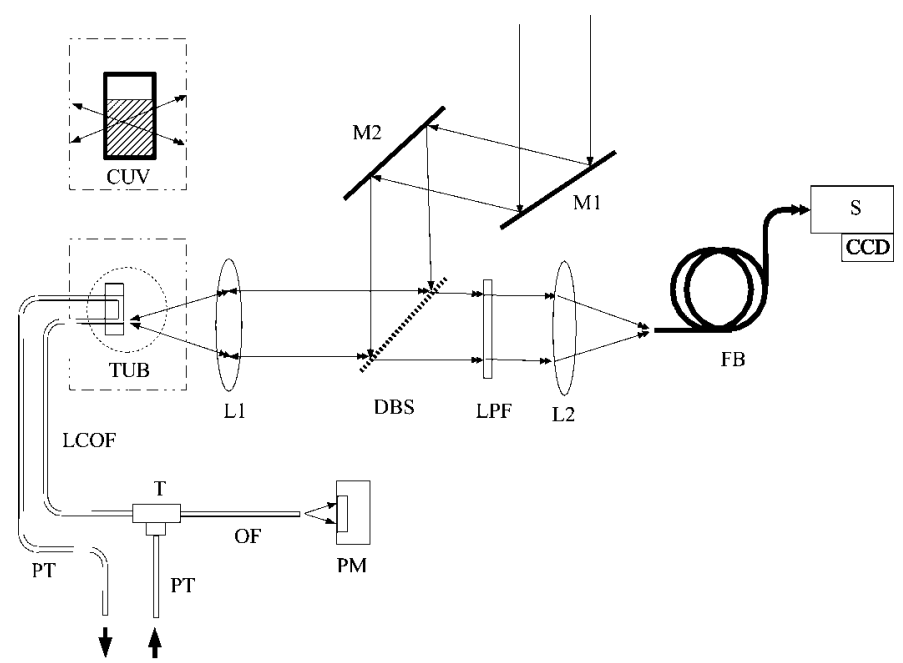

FIG. 1. Optical system for delivering and collecting light from two liquid containers: LCOFs and standard cuvettes. The dashed rectangles indicate the two different holders that can be placed at the focus of lens L1. (M1, M2) mirrors; (DBS) dichroic beamsplitter; (L1, L2) lenses; (CUV) cuvette; (TUB) tubing holder system (shown in detail in next figure); (LCOF) liquid core optical fiber; (PT) plastic tubing; (T) tconnector; (OF) optical fiber; (PM) power meter; (LPF) long-pass filter; (FB) fiber bundle; and (S) spectrograph. 


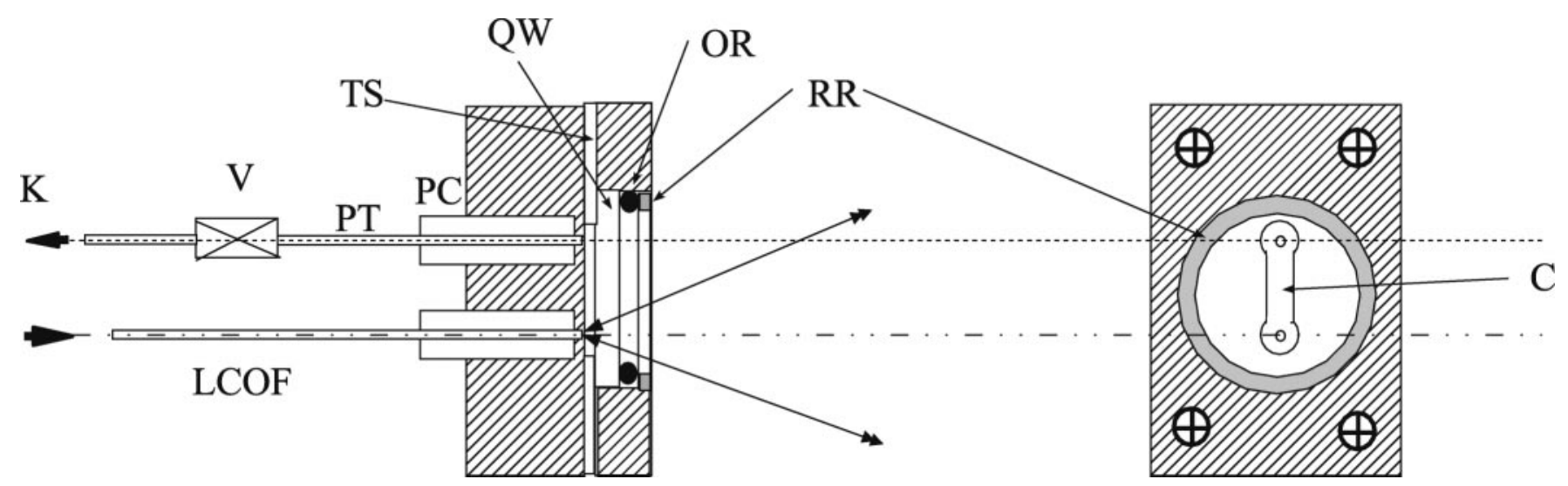

FIG. 2. Housing for the LCOF. (RR) retaining ring; (OR) O-ring; (QW) quartz window; (TS) Teflon sheet; (PC) plastic chuck; (V) valve; and (C) channel dug in the Teflon sheet to guide liquid from LCOF to output tube.

Angle-Dependent Loss Model. The model just described can be generalized by noting that attenuation is a function of propagation angle, due to the different numbers of reflection events..$^{9-12}$ In this model, when a light ray encounters the core-cladding interface, a fraction $R$ $(\leq 1)$ undergoes perfect specular reflection and the remainder is immediately lost (i.e., sent in directions that cannot reach the detector) due to diffuse scattering and other non-absorptive mechanisms. We call this quantity $R$ the overall reflection efficiency. The simplest approximation is to treat $R$ as a constant over the experimental range of propagation angles and wavelengths. A meridional ray of light traversing a length $z$ along the waveguide axis at propagation angle $\theta$ will thus attenuate according to

$$
T(z, \theta)=e^{-\mu_{\mathrm{a}} \cdot(z / \cos \theta)} R^{N} 2 \approx e^{-\left(\mu_{\mathrm{a}}-\theta \ln R / d\right) z}=e^{-\delta(\theta) z}
$$

where $d$ is the inner diameter of the waveguide, $N=z /$ $(d \cot \theta)$ is the number of reflections experienced, $\mu_{\mathrm{a}}$ is the (wavelength-dependent) absorption coefficient, and $\delta(\theta) \equiv e^{-\left(\mu_{\mathrm{a}}-\theta \ln R / d\right)}$ is the extinction coefficient, now having separate dependences on absorption and reflection.

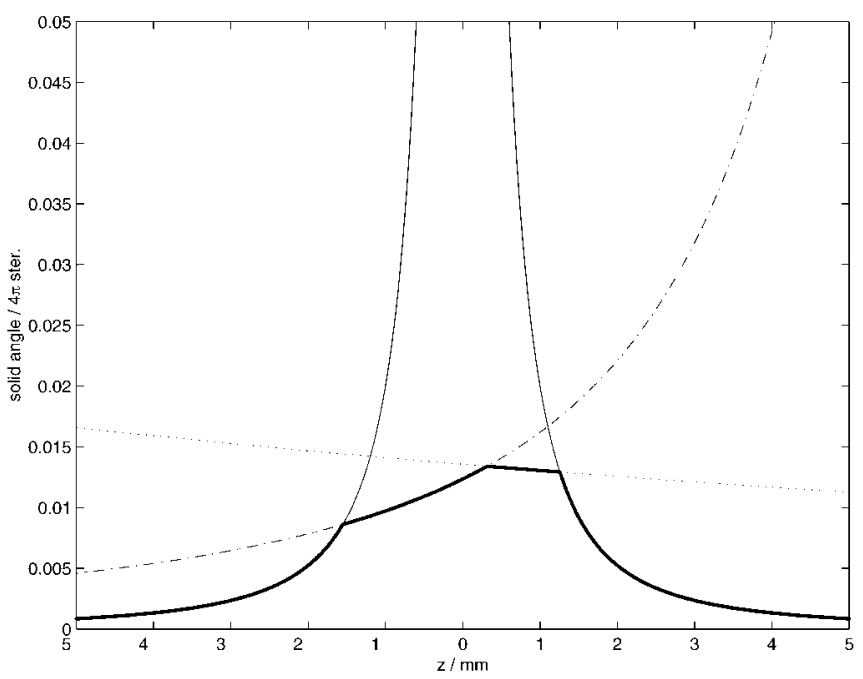

FIG. 3. Depth dependence of the acceptance angle for the collection system (two lenses and the optical fiber bundle). The thinner lines show the angles $\gamma_{\mathrm{FB}}$ (solid), $\gamma_{\mathrm{L} 1}$ (dash), and $\gamma_{\mathrm{L} 2}$ (dot-dash) for the individual components, as described by Eq. 1. The heavy black line shows the acceptance angle of the composite system.
Equation 3 can thus be generalized by integrating over the incident angle $\theta$ and Raman emitted angle $\gamma$, yielding

$$
p_{\mathrm{R}}(z)=\int_{\theta=0}^{\theta_{0}} \int_{\gamma=0}^{\gamma_{0}} p_{\mathrm{E}}(\theta) \cdot e^{-\left[\delta_{1}(\theta)+\delta_{2}(\gamma)\right] z} \cdot 2 \pi s \gamma \mathrm{d} \gamma \mathrm{d} \theta
$$

Here $p_{\mathrm{E}}$ is the excitation power per unit angle $\left(\int p_{\mathrm{E}}(\theta) \cdot \mathrm{d} \theta\right.$ $\left.=P_{0}\right)$, and $2 \pi \gamma \mathrm{d} \gamma$ is the differential solid angle of Raman emission.

When the angle-dependence of $\delta$ is negligible, then this theory should be identical to the simple mono-exponential decay model. Equation 6 reduces to

$$
\begin{aligned}
p_{\mathrm{R}}(z) & =2 \pi s\left[\int_{\theta=0}^{\theta_{0}} p_{\mathrm{E}}(\theta) \mathrm{d} \theta\right]\left[\int_{\gamma=0}^{\gamma_{0}} \gamma \mathrm{d} \gamma\right]\left[e^{-\left(\delta_{1}+\delta_{2}\right) z}\right] \\
& =P_{0} s \pi \gamma_{0}^{2}\left[e^{-\delta_{1} z} \cdot e^{-\delta_{2} z}\right]
\end{aligned}
$$

which reproduces Eq. 3 with mono-exponential transmission efficiencies $T_{1}(z)=e^{-\delta_{1} z}$ and $T_{2}(z)=e^{-\delta_{2} z}$, as expected.

Raman Collection from Cuvette. The cuvette similarly presents a pathlength $w$ to the excitation beam. Modeling the light propagation within the cuvette is easier than for the waveguide, because there are no internal reflection losses $\left(\delta=\mu_{\mathrm{a}}\right)$. However, instead of all Raman signal being guided to a common aperture at $z=0$, now the Raman emission at different depths is collected over different acceptance angles $\gamma(z)$, according to Eq. 2. The Raman power per unit depth delivered to the fiber bundle is thus given by

$$
p_{\text {cuv }}(z)=\int_{\gamma=0}^{\gamma_{\mathrm{sys}}(z)}\left[P_{0} e^{-\mu_{\mathrm{a} 1 z^{\prime}}}\right] e^{-\mu_{\mathrm{a} 2} z^{\prime}} \cdot 2 \pi s \gamma \mathrm{d} \gamma
$$

where $z^{\prime} \equiv z+w / 2$ is introduced because the cuvette is centered at $z=0$. Note that the depth-dependent acceptance angle appears in the upper limit of integration.

Strictly, this equation approximates the focused excitation light as a pencil beam along the $z$ axis with a negligible diameter. This overestimates the collected signal, because off-axis Raman scattering is not collected as efficiently. We find the approximation acceptable in this case, however, because (1) near the focal plane $(z=0)$, the focused beam's diameter is sufficiently small (160 $\mu \mathrm{m}$, calculated from measurements in air) that off-axis 
effects are negligible; and (2) at other depths, despite the larger beam diameter, even the on-axis collection efficiency drops so steeply that contributions to the total signal are very small.

Enhancement Ratio. Equation 7 resembles Eq. 6 for the waveguide, and the similarity can be emphasized by writing the latter equation as

$$
p_{\mathrm{wv}}(z)=\int_{\gamma=0}^{\gamma_{0}}\left[\int_{\theta=0}^{\theta_{0}} p_{\mathrm{E}}(\theta) \cdot e^{\delta_{1}(\theta) z} \mathrm{~d} \theta\right] e^{-\delta_{2}(\gamma) z} \cdot 2 \pi s \gamma \mathrm{d} \gamma
$$

The parallel forms emphasize that we can compare measurements taken in the two geometries. Note that the upper limit of integration for $\gamma$ in Eq. 8 is not depth-dependent, unlike Eq. 7 for the cuvette.

Equations 7 and 8 describe the power per unit sample depth delivered to the fiber bundle input face. The waveguide enhancement factor $E$ is thus given by

$$
E=\frac{\int_{0}^{L} p_{\mathrm{wv}}(z) \mathrm{d} z}{\int_{-w / 2}^{w / 2} p_{\mathrm{cuv}}(z) \mathrm{d} z}
$$

In Eq. 9, we assume that all downstream losses due to the fiber bundle, spectrograph, and CCD array detector are common to the two geometries and will not affect the enhancement ratio. This assumes that the fibers in the bundle accept light with equal efficiency and deliver their light to the CCD with equal efficiency, and that dead space losses between fibers affect the waveguide and cuvette measurements equally. We find these assumptions to be valid for this system. In particular, both geometries illuminate a multi-fiber sub-region of the collection bundle and therefore experience similar dead-space losses. Note that, because the bundle transforms to a linear array at the entrance to the spectrograph, variations in how the input face is filled do not affect the final spectral resolution. Angle-dependent variations in Fresnel reflection coefficients were also found to have negligible effects upon the calculated enhancement ratio; both systems have the same set of refractive index mismatches (aqueous/quartz and quartz/air).

\section{MEASUREMENTS}

Determination of Waveguide Reflection Efficiency. The reflection efficiency $R$ can be measured by inserting an optical fiber into the end of the waveguide, translating it, and measuring the power transmitted as the distance $z$ to the far end is varied.7,10,11,13 According to the theory above, the fraction of power transmitted should be

$$
\frac{p_{\text {out }}(z)}{p_{\text {in }}}=\frac{\int p_{\mathrm{E}}(\theta) \cdot e^{-\delta_{1}(z)} \mathrm{d} \theta}{P_{0}}=e^{-\mu_{\mathrm{a} 1} z}\left\langle e^{\theta(\ln R / d) z}\right\rangle
$$

where \langle\rangle denotes a weighted average over all of the input light. Different distributions of input light will have different averages. For the case of light launched uniformly into the emission solid angle of the fiber, $p_{\mathrm{E}}(\theta) \propto \theta$. Based upon observations of the light cone emerging from the optical fiber in air, we assume that this angular distribu-

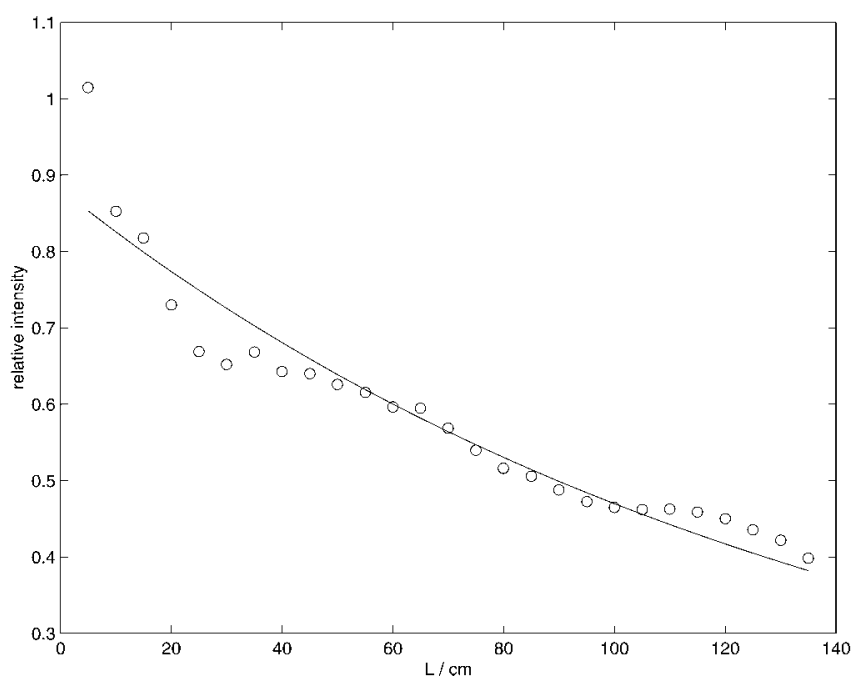

FIG. 4. Measuring the internal reflection coeffecient $R$ of the waveguide. (Circles) measured values of $P_{\text {out }} / P_{\text {in }}$; (solid line) best fit using Eq. 11, with $R=0.966$.

tion is a sufficient first-order approximation for the light emerging from the multimode fiber within an aqueous medium. In this case, Eq. 10 evaluates to

$$
\begin{aligned}
& \frac{P_{\text {out }}(z)}{P_{\text {in }}} \\
& \quad=e^{-\mu_{\mathrm{a} 1} z} \cdot \frac{2}{\left[(\ln R / d) \theta_{0} z\right]^{2}}\left\{e^{(\ln R / d) \theta_{0} z}\left[\left(\ln \frac{R}{d}\right) \theta_{0} z-1\right]+1\right\}
\end{aligned}
$$

Measured values of $P_{\text {out }} / P_{\text {in }}$ at different depths $z$ were obtained as follows. An optical fiber $(100 \mu \mathrm{m}$ inner, 250 $\mu \mathrm{m}$ outer diameter) delivering $830 \mathrm{~nm}$ light was inserted into the water-filled waveguide and moved to different positions. The NA of the fiber emission into water was calculated to be 0.165 , corresponding to $\theta_{0}=9.5^{\circ}$ in the equations above. A plot of the measured extinctions is given in Fig. 4, along with the best fit using Eq. 11. The model gives $R=0.966 \pm 0.003(90 \%$ confidence interval). Because the datapoints are highly reproducible, we attribute the fine structure to local rough spots or other inhomogeneities within the waveguide.

The model fit in Fig. 4 looks approximately like a simple exponential decay. Indeed, the data can be modeled just as accurately using a single exponential, with a decay coefficient of $\delta_{1}=0.0086 \mathrm{~mm}^{-1}$. We emphasize, however, that unlike $R$, this is not an intrinsic property of the system. Rather, $\delta$ depends upon the distribution of angles at which light is launched into the waveguide. Using angle-dependent theory is essential for extrapolation to other measurements in which the illumination of the waveguide is substantially different (as will be discussed below).

Measurement of Raman Backscatter versus Waveguide Length. The dependence of Raman signal upon waveguide length $L$ was investigated. Raman backscattering spectra were gathered from a $20 \%$ aqueous ethanol solution that was placed in the waveguide. Prior to the experiment, the absorption spectrum of the solution was measured on a spectrophotometer, permitting calculation of $\mu_{\mathrm{a}}$ values at excitation and various emission wave- 


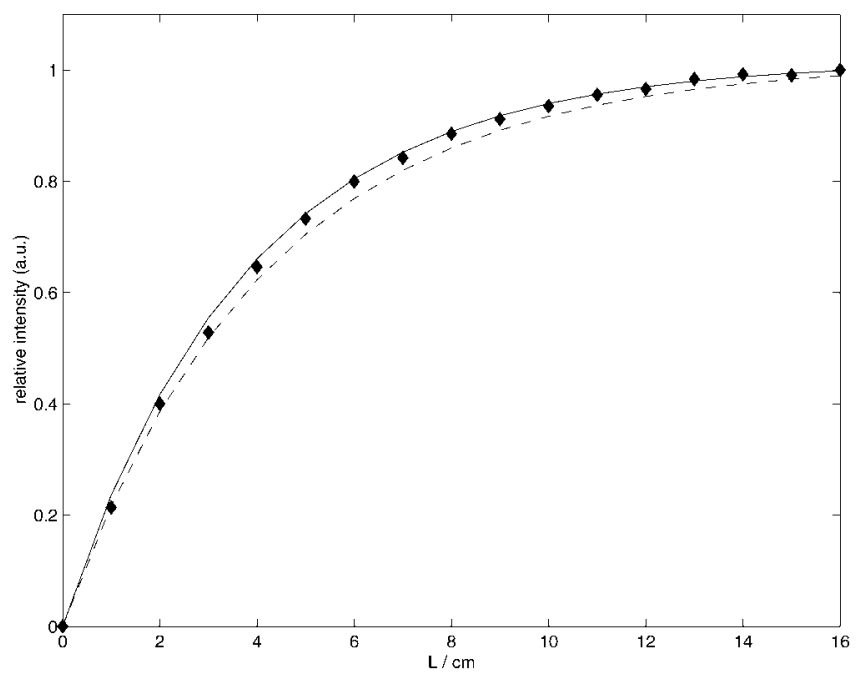

FIG. 5. $\quad 888 \mathrm{~cm}^{-1}$ Raman scattering signal from $20 \%$ ethanol, measured for different waveguide lengths $L$ as described in the text. (Diamonds) measured values. (Solid curve) prediction using angle-dependent theory. (Dashed curve) prediction using mono-exponential theory.

lengths. The useful length $L$ of the waveguide was varied via advancing, from the opposite end, the same optical fiber mentioned in the previous section, thereby obstructing the remainder of the waveguide. The relative strength of the ethanol Raman band at $888 \mathrm{~cm}^{-1}$ is plotted in Fig. 5 , normalized to 1 at its largest value. The plot has been corrected for signal contributions from the $z>L$ regime (due to the optical fiber not completely filling the waveguide).

Also shown in the figure are two theoretical models for the dependence of Raman signal upon $L$. The monoexponential prediction of Eq. 4, based upon $\delta_{1}=0.0086$ $\mathrm{mm}^{-1}$ as derived from the previous section, is plotted as a dashed line. The angle-dependent prediction, from integration of $p_{\mathrm{wv}}(z)$ from Eq. 8, is plotted as a solid line. In the latter prediction, $p_{\mathrm{E}}(\theta) \propto \theta$ was again used, this time based upon power measurements of the excitation beam.

Both theoretical plots reproduce the basic shape of the experimental curve. This result is consistent with that of Altkorn et al., who used a mono-exponential decay model to fit data from a forward-scattering waveguide geometry. ${ }^{5}$ The fit is noticeably better, however, using the angledependent theory. We stress that mono-exponential theory could model the data just as accurately, but not using $\delta$ values consistent with the plot of Fig. 4 .

Measurement of Enhancement Factor. The waveguide Raman enhancement factor $E$ of Eq. 9 was measured experimentally. A $20 \%$ ethanol solution was placed in (a) the waveguide, with $L=17 \mathrm{~cm}$, and (b) the cuvette, with $w=1 \mathrm{~cm}$. Spectra of six separate sample insertions were obtained for each of the two geometries, for 160 seconds each. Representative spectra measured using the waveguide and cuvette geometries are shown in Fig. 6, with the latter scaled by a factor of 10 to facilitate comparison. The strong peaks are all due to the ethanol; some small Raman features due to the Teflon ${ }^{\circledR}-\mathrm{AF}$ are visible below $800 \mathrm{~cm}^{-1}$. Areas under the five most prominent ethanol Raman peaks $(888,1058,1097,1287$, and 1463

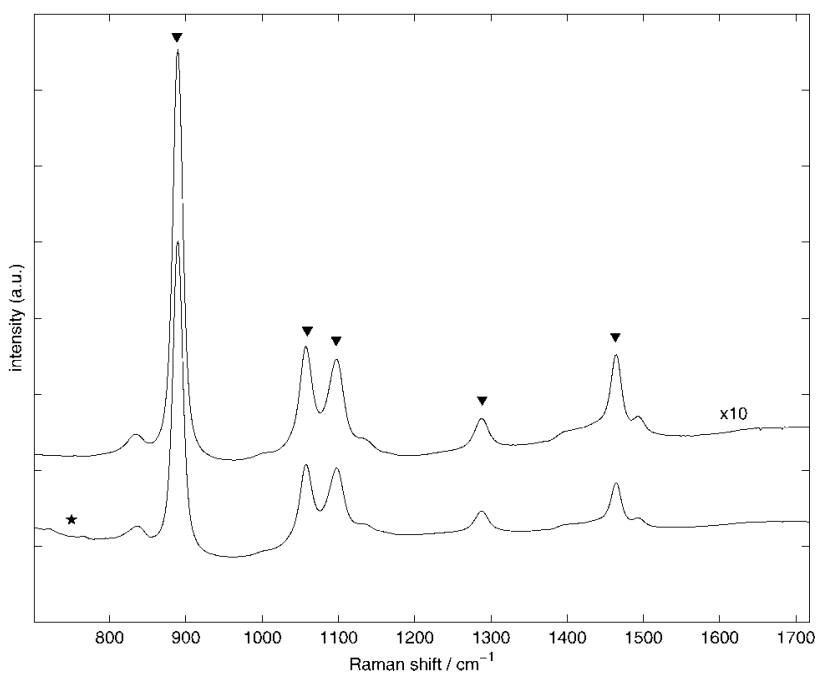

FIG. 6. Spectra of $20 \%$ aqueous ethanol, measured using the waveguide (lower) and cuvette (upper, multiplied $\times 10$ ) geometries. System background and autofluorescence have been subtracted, and spectra are offset for clarity. The triangles indicate the five peaks for which enhancement was calculated. The star indicates the spectral region in which peaks from the waveguide material can be discerned.

$\mathrm{cm}^{-1}$ ) were calculated. From these area measurements, enhancements $E$ at those wavenumbers were calculated.

The angle-dependendent light propagation model was employed to predict the enhancement of collected Raman signal. Figure 7 plots the Raman signals, $p_{\mathrm{wv}}$ and $p_{\mathrm{cuv}}$, arriving from various depths $z$, as predicted by Eqs. 8 and 7, respectively, for the $888 \mathrm{~cm}^{-1}$ Raman band. The dashed curve, corresponding to the cuvette, is a compressed plot of data similar to that of Fig. 3. As expected, the waveguide geometry explores a much greater collection depth.

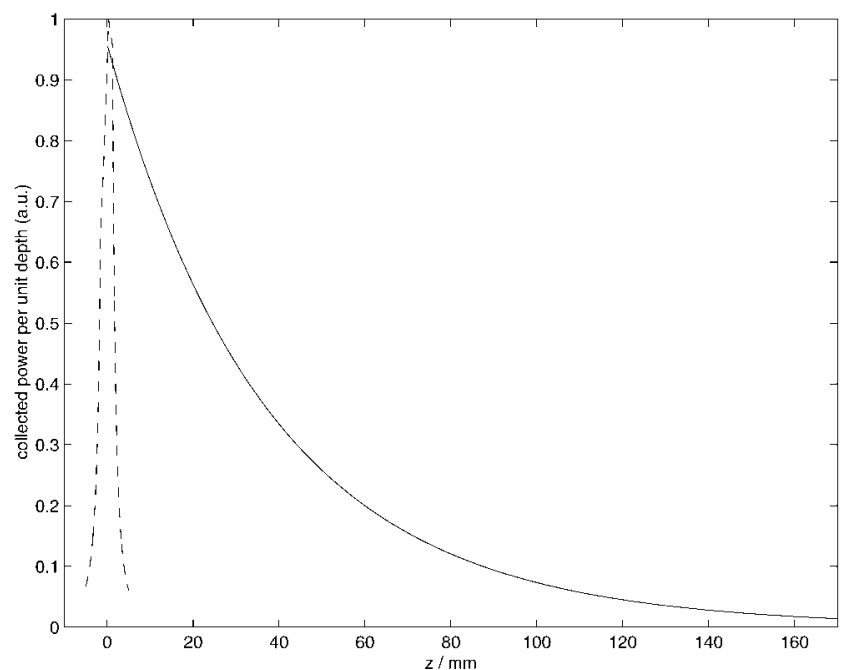

FIG. 7. Predicted Raman power per unit depth $z$ collected from the aqueous samples, for the $888 \mathrm{~cm}^{-1}$ transition ( $895 \mathrm{~nm}$ emission). (Solid curve) $p_{\mathrm{wv}}$ (waveguide), given by Eq. 8 ; (dashed curve) $p_{\text {cuv }}$ (cuvette), given by Eq. 7. The vertical axis is scaled to unity for collection of scattering from the $z=0$ plane, for which the two geometries are nearly equally efficient. The total Raman signal enhancement is the ratio of the areas under these curves. Note that the cuvette curve has values from $-5 \mathrm{~mm} \leq z \leq 5 \mathrm{~mm}$, since the focus is at the center of the cuvette, whereas the waveguide curve has values from $0 \leq z \leq 170 \mathrm{~mm}$ (the length of the waveguide, with the output face at $z=0$ ). 


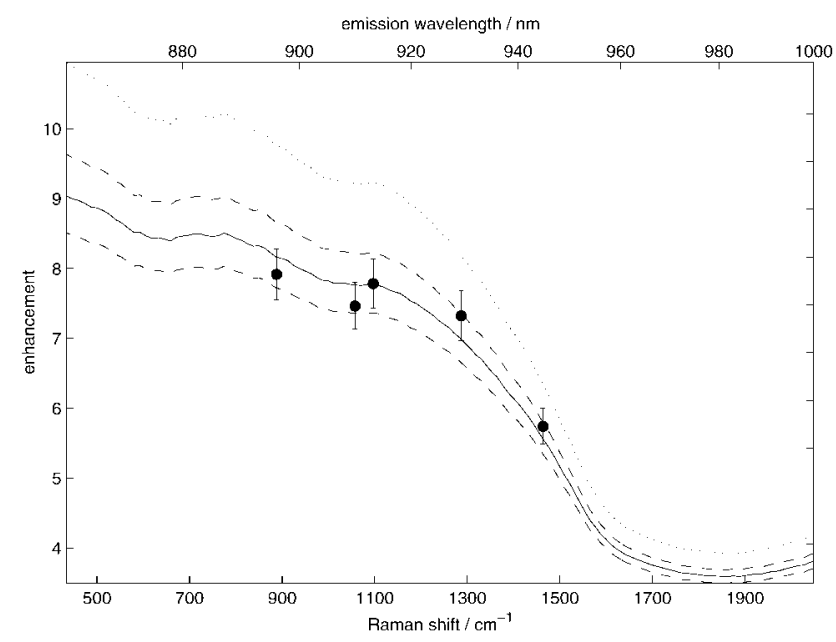

FIG. 8. Predicted and measured Raman enhancements for the geometries studied in this paper. (Solid dots with error bars) experimental measurements. (Solid line) prediction using angle-dependent propagation theory, with $R=0.966$. (Dashed lines) uncertainty in theoretical prediction due to experimental measurement of $R$, described earlier. (Dotted line) prediction using mono-exponential propagation theory, using $\delta_{1}=0.0086 \mathrm{~mm}^{-1}$ for excitation light as determined from Fig. 4; in this case the agreement with experiment is poor. The lower $\mathrm{x}$ axis gives the Raman shift, while the upper $\mathrm{x}$ axis gives the emission wavelength for excitation at $830 \mathrm{~nm}$.

The ratio of the areas under the curves in Fig. 7 is the Raman enhancement factor, $E$, as given by Eq. 9. As a function of emission wavelength, these factors are plotted in Fig. 8. A $90 \%$ confidence interval, due to uncertainty in the measurement of $R$, is indicated by dashes. There is a noticeable decrease at larger wavelengths, due to water absorption peaking at $980 \mathrm{~nm}$. The experimentally measured enhancements are also shown, plotted as circles, with error bars due to the multiple measurements in each geometry. Agreement between theory and experiment is seen.

Also shown in the plot are predictions of $E$ based upon the mono-exponential theory of Eq. 4, using extinction coefficients $\delta_{1}$ and $\delta_{2}$ derived from the data of Fig. 4 and the measured $\mu_{\mathrm{a}}$ values of $20 \%$ aqueous ethanol. Unlike the plot of Fig. 5, which was modeled fairly well, here the mono-exponential theory noticeably overestimates the observed enhancements. This happens because the extinction factor $\delta$ was calculated using a narrower cone of angles than in the Raman geometry. Light rays at higher angles endure more reflections and greater losses, leading to a lower Raman signal than predicted using $\delta$.

\section{DISCUSSION}

The field of waveguide-enhanced aqueous Raman spectroscopy is still in its infancy, especially for biomedical applications. One limiting factor is that techniques for manufacturing high-quality optical waveguides from Teflon ${ }^{\circledR}-\mathrm{AF}$ are not yet optimized. As new waveguides are produced, they need to be tested. Based upon the tests just described, we suggest that measuring the effective reflection coefficient $R$ is a useful number for characterizing such waveguides. Although light propagation can be more carefully modeled using mode theory, ${ }^{10-12}$ a raybased model with a constant $R$ appears sufficient for predicting several key aspects of the waveguides' perfor-
TABLE II. Predicted enhancement values for various combinations of reflectivity and excitation wavelength. For visible excitation and high reflectivity, the enhancement approaches two orders of magnitude, in agreement with published reports of similar geometrical comparisons.

\begin{tabular}{ccc}
\hline$\lambda_{\text {ex }}(\mathrm{nm})$ & $R=0.966$ & $R=0.995$ \\
\hline 830 & 9 & 16 \\
785 & 11 & 22 \\
632.8 & 17 & 65 \\
514.5 & 18 & 82 \\
\hline
\end{tabular}

mance in Raman spectroscopy. If both $R$ and the range of launching angles are specified, then the waveguide loss in $\mathrm{dB} / \mathrm{m}$ can be calculated.

Significantly, the $R$-based model recognizes that light in the waveguide propagates more efficiently at smaller angles. In our present waveguide, where $R$ was noticeably less than 1, modeling this effect was important. As shown in Fig. 8, a simpler, mono-exponential theory of light attenuation was unsuccessful in predicting the observed strength of the Raman signals from the waveguide. Separate measurements of waveguide transmission efficiency also exhibited clear angle dependence consistent with theory (data not shown).

It is evident that the observed reflection efficiency of 0.966 is far from optimal. Improving the reflection efficiency can significantly increase the signal collection. We calculate, using the model presented, that an upgrade to $R=0.995$ would increase the Raman signal strength at $888 \mathrm{~cm}^{-1}$ by a factor of two. In addition to improved fabrication of the waveguides themselves, it is possible to improve reflectivity by additional coating of the inside walls, for example with poly(allylamine hydrochloride) and poly(acrylic acid) as described by Inya-Agha et al. ${ }^{14}$ We note that, in the limit of high reflectivity, light attenuation becomes asborption-dominated and mono-exponential theory becomes sufficient to predict the Raman enhancement factor.

The $830 \mathrm{~nm}$ excitation used in these studies is not optimal for samples whose $\mu_{\mathrm{a}}$ values are dominated by water, which has an absorption peak at $980 \mathrm{~nm}$. We chose $830 \mathrm{~nm}$ excitation because we anticipate applications to urine, whose absorption spectrum exhibits a minimum around this wavelength due to the combined effects of water and bilirubin. For other aqueous samples of biomedical interest, e.g., blood serum or saliva, it might be more advantageous to move to shorter wavelengths. Table II lists the predicted Raman enhancement advantages using various excitation wavelengths and reflection efficiencies, assuming a 2-meter-long waveguide and 1-cmthick cuvette. Assumed values of $\mu_{\mathrm{a}}$ were taken from the water data of Kou et al. ${ }^{15}$ Enhancements of two orders of magnitude are seen in the best cases, in qualitative agreement with published results (see Table I); exact comparison is precluded because the precise cuvette/vial geometries were not described. Note that the enhancements in Table II have nothing to do with the $\sim \lambda^{-4}$ scaling of the Raman scattering cross-section, which provides an additional benefit of moving to shorter excitation wavelengths.

On a practical level, the ability to compare cuvette and waveguide geometries quantitatively is valuable. The cuvette is much easier and more robust to align than the 
waveguide. As shown in Fig. 1, it is easy to make the two geometries exchangeable. Thus, one can align the rest of the optical system using the cuvette and establish a baseline Raman signal strength. The model presented above can then calculate the expected signal increase within the waveguide, providing a target value during its alignment.

The demonstrated enhancement in signal should mean that dissolved chemicals can be detected more accurately or more rapidly. Verification of this prediction will be the subject of a future communication.

In summary, we have presented an extension to existing theory that helps us to explain observed data in waveguide enhancement of Raman signals. The theory is particularly important when reflection efficiency, rather than absorption, limits the useful length of a waveguide. Although the waveguide studied here had a reflection efficiency less than 0.97 and the excitation wavelength was not optimal for samples with $\mu_{\mathrm{a}}$ values close to those of water, the amount of Raman enhancement obtained is consistent with published values from other studies. Even under the present conditions, the signal enhancement factor was greater than 5 at all wavelengths, while using a fraction of the sample volume required for a $1-\mathrm{cm} \mathrm{cu}-$ vette. We anticipate that waveguide-enhanced Raman spectroscopy will play a significant role in chemical analysis of clear aqueous biological samples in the near future.

\section{ACKNOWLEDGMENTS}

We thank Ilia Koev and Biogeneral, Inc., for providing a sample of Teflon ${ }^{\circledR}$-AF waveguide tubing, and the DuPont company for permission to conduct these studies. Funding for this research was provided by the Whitaker Foundation, award RG-02-0079.

1. G. W. Walrafen and J. Stone, Appl. Spectrosc. 26, 585 (1972).

2. L. Song, S. Liu, V. Zhelaskov, and M. A. El-Sayed, Appl. Spectrosc. 52, 1364 (1998).

3. M. J. Pelletier and R. Altkorn, Anal. Chem. 73, 1393 (2001).

4. B. J. Marquardt, P. G. Vahey, R. E. Synovec, and L. W. Burgess, Anal. Chem. 71, 4808 (1999).

5. R. Altkorn, I. Koev, and M. J. Pelletier, Appl. Spectrosc. 54, 1169 (1999).

6. M. Holtz, P. K. Dasgupta, and G. Zhang, Anal. Chem. 71, 2934 (1999).

7. R. Altkorn, I. Koev, R. P. V. Duyne, and M. Litorja, Appl. Opt. 36, 8992 (1997).

8. R. Altkorn, M. D. Malinsky, R. P. van Duyne, and I. Koev, Appl. Spectrosc. 55,373(2001).

9. H. Blumenfeld, E. Gaillard, and P. Rebourgeard, Nucl. Instrum. Methods Phys. Res., A 309, 169 (1991).

10. P. Dress and H. Franke, Appl. Phys. B 63, 12 (1996).

11. P. Dress and H. Franke, Rev. Sci. Instrum. 68, 2167 (1997).

12. W. Wei, H. Qushe, W. Tao, F. Minhao, L. Yuanmin, and R. Gouxia, Anal. Chem. 64, 22 (1992).

13. R. Altkorn, I. Koev, and A. Gottlieb, Appl. Spectrosc. 51, 1554 (1997).

14. O. Inya-Agha, S. Stewart, T. Veriotti, M. L. Bruening, and M. D. Morris, Appl. Spectrosc. 56, 574 (2002).

15. L. H. Kou, D. Labrie, and P. Chylek, Appl. Opt. 32, 3531 (1993). 